\title{
Baixo conhecimento de doadores de sangue sobre a hanseníase como fator de vulnerabilidade para a disseminação da doença
}

\author{
Low knowledge of blood donors about leprosy as a vulnerability factor for the spread \\ of the disease
}

Regina Rafael Teixeira ${ }^{1}$, Tamires de Oliveira Santos ${ }^{1}$, Amanda Aparecida Silva de Aguiar ${ }^{2}$, Thaís Batista de Carvalho ${ }^{3}$, Elaine Cristina Negri ${ }^{4}$ Eliana Peresi Lordelo ${ }^{5^{*}}$

\begin{abstract}
${ }^{1}$ Bacharel em Biomedicina pela Universidade do Oeste Paulista - Unoeste; ${ }^{2}$ Mestranda do Curso de Mestrado em Ciências da Saúde da Universidade do Oeste Paulista - Unoeste; ${ }^{3}$ Doutora em Doenças Tropicais pela Faculdade de Medicina de Botucatu - UNESP, Docente do Curso de Biomedicina da Universidade do Oeste Paulista - Unoeste; ${ }^{4}$ Doutora em Ciências pela Escola de Enfermagem de Ribeirão Preto da Universidade de São Paulo - USP, Docente do Curso de Enfermagem da Universidade do Oeste Paulista, Unoeste; ${ }^{5}$ Doutora em Doenças Tropicais pela Faculdade de Medicina de Botucatu - UNESP, Docente do Curso de Mestrado em Ciências da Saúde da Universidade do Oeste Paulista - Unoeste
\end{abstract}

\begin{abstract}
Resumo
Objetivo: caracterizar o doador de sangue e seu conhecimento sobre a hanseníase, visando contribuir para identificar pontos de vulnerabilidade sobre a doença. Metodologia: foram entrevistados doadores de sangue $(n=199)$ através de um questionário estruturado abordando características socioeconômicas e o conhecimento sobre a hanseníase. Para a análise dos dados foi utilizado o método de Goodman e considerado significativo $p<0,05$. Resultados: dentre as perguntas sobre a hanseníase, a maioria dos participantes $(65,83 \%)$ não tinha conhecimento da doença e nem o seu modo de transmissão $(75,88 \%)$ e quando computado o conhecimento da Hanseníase, 1,51\% conheciam, 39,70\% conheciam pouco e 58,79\% não conheciam a doença. Nossos resultados demonstraram que somente a escolaridade teve associação significativa com a falta de conhecimento sobre a hanseníase $(p=0,0273)$. Conclusão: verificou-se déficit de conhecimento da população geral quanto à hanseníase. Sugerimos um aprimoramento da divulgação das informações quanto à doença a fim de promover melhoras nos serviços de saúde, acompanhamento dos doentes e prevenção da população saudável.
\end{abstract}

Palavras-chave: Hanseníase. Doador de Sangue. Conhecimento.

\begin{abstract}
Objective: to characterize the blood donor and his knowledge about leprosy, aiming to contribute to identify vulnerability points about the disease. Methodology: blood donors ( $n=199)$ were interviewed through a structured questionnaire addressing socioeconomic characteristics and knowledge about leprosy. For the data analysis, the Goodman method was used and considered significant $p<0.05$. Results: Among the questions about leprosy, most participants (65.83\%) did not know about the disease and its mode of transmission (75.88\%) and when computing the knowledge of leprosy, 1.51\% knew, 39,70\% knew little and 58.79\% did not know the disease. Our results showed that only schooling had a significant association with the lack of knowledge about leprosy $(p=0,0273)$. Conclusion: there was a lack of knowledge of the general population regarding leprosy. We suggest an improved dissemination of information about the disease to promote improvements in health services, patient monitoring and prevention of the healthy population. Keywords: Leprosy. Blood donnor. Knowledge.
\end{abstract}

\section{INTRODUÇÃO}

A hanseníase é uma doença infectocontagiosa, de evolução crônica, causada pelo Mycobacterium leprae ou bacilo de Hansen. A doença atinge pele e nervos periféricos, podendo levar a sérias incapacidades físicas. ${ }^{(1)}$ Quando não diagnosticada e tratada adequadamente, é capaz de evoluir para deformidades, influenciando em problemas sociais, como o preconceito aos doentes. ${ }^{(2)}$

Correspondente/Corresponding: *Eliana Peresi-Lordelo-Universidade do Oeste Paulista - Unoeste - End: Rod. Raposo Tavares km 572, CEP 19067-175 Presidente Prudente - SP - Brazil - Tel: (18) 3229-1289 Email: elianaperesi@yahoo.com.br
Atualmente, esta doença representa um grave problema de saúde pública para o Brasil e em países em desenvolvimento, e apesar de todo o empenho para sua eliminação no ano 2014, 31.064 novos casos foram notificados no país, dentre estes, 14.109 são mulheres e 2.341 são crianças, ficando atrás apenas da Índia, com 125.785 novos casos. ${ }^{(3)}$ De acordo com o Ministério da Saúde (2014), cinco estados mostraram prevalência acima de três casos por grupo de 10 mil habitantes (Mato Grosso, Tocantins, Maranhão, Pará e Rondônia). Embora o estado de São Paulo tenha apresentado um coeficiente de detecção de 0,34 por 10 mil habitantes, em 2015 na 
região de Presidente Prudente foram registrados 56 casos de pacientes por residência. ${ }^{(4,5)}$

O contágio dá-se de pessoa a pessoa e está relacionado a fatores socioeconômicos, tais como: a condição de moradia da população, alimentação e higiene. Por isso, associada a desigualdades sociais, afeta principalmente as regiões mais carentes do mundo. ${ }^{(6)} \mathrm{O}$ tratamento é feito através de uma combinação de fármacos seguros e eficazes, evitando o surgimento de resistência aos medicamentos. Apesar de haver a cura, a terapia é frequentemente abandonada, o que leva a disseminação da doença. ${ }^{(2,7)}$

Baseado na natureza crônica da doença e a dinâmica da transmissão, as mortes por hanseníase continuarão a ocorrer durante décadas, tornando relevante o processo de educação em saúde e a abordagem desta doença de grande ênfase social. ${ }^{(8)}$

O desconhecimento da população geral sobre a hanseníase ainda é muito grande. A deficiência no conhecimento sobre a doença geralmente acarreta demora no diagnóstico e tratamento, colaborando para o acréscimo do número de indivíduos infectados assim como as sequelas permanentes. Por outro lado, já foi demonstrado que ações educativas têm efeito informativo, contribuindo para o aumento do conhecimento da doença. ${ }^{(9)}$ Desta forma, o objetivo do trabalho foi a caracterização do doador de sangue e de seu conhecimento sobre a hanseníase, visando contribuir para identificar pontos de vulnerabilidade sobre a doença.

\section{METODOLOGIA}

Foram entrevistados doadores de sangue do Hemocentro da Santa Casa de Presidente Prudente/SP, aos quais foi aplicado um instrumento com questões estruturadas que determinou a sua caracterização quanto idade, sexo, estado civil, religião, escolaridade e condição socioeconômica ${ }^{(10,11)}$; e o conhecimento sobre a hanseníase. A avaliação do conhecimento sobre a hanseníase foi elaborada pelos pesquisadores baseada nas características de transmissão, sintomatologia, tratamento e prevenção da doença. Os participantes foram divididos em grupos de acordo com suas repostas: conhece (6-8 respostas corretas); conhece pouco (4-5 repostas corretas); e não conhece (0-3 respostas corretas). 0 método de Goodman foi utilizado com a finalidade de verificar se ocorre diferença entre as proporções multinomiais, fixando a variável multinomial de interesse e avaliando os contrastes das proporções das classes dessa população. ${ }^{(12)}$ Este trabalho foi aprovado pelo Comitê de Ética e Pesquisa (CEP) (CAAE: 54884116.0.0000.5515) e pela Coordenadoria de Pesquisa, Desenvolvimento e Inovação (CPDI: 3117), ambos da Universidade do Oeste Paulista - UNOESTE.

\section{RESULTADOS}

Participaram da pesquisa 199 doadores de sangue, todos aprovados pela triagem de doação. Dentre as perguntas sobre a hanseníase, a maioria $(65,83 \%)$ não sabia sobre a doença e nem o seu modo de transmissão (75,88\%). Entretanto, questionados sobre a percepção sobre campanhas sobre a doença, a maioria relatou já ter visto $(66,33 \%)$. Quando computado o conhecimento da hanseníase, 1,51\% conheciam, 39,70\% conheciam pouco e $58,79 \%$ não conheciam a doença (Tabela 1 ).

Tabela 1 - Questões e respostas sobre a hanseníase e o conhecimento do doador de sangue.

\begin{tabular}{|c|c|c|c|}
\hline Questões sobre a hanseníase & & $\mathrm{n}$ & $\%$ \\
\hline \multirow[t]{2}{*}{ Sabe o que é hanseníase? } & Não sabe & 131 & 65,83 \\
\hline & Sabe & 68 & 34,17 \\
\hline \multirow[t]{2}{*}{ Como a hanseníase é transmitida? } & Não sabe & 151 & 75,88 \\
\hline & Sabe & 48 & 24,12 \\
\hline \multirow[t]{2}{*}{ Quais são os sintomas da hanseníase? } & Não sabe & 98 & 49,25 \\
\hline & Sabe & 101 & 50,75 \\
\hline \multirow[t]{2}{*}{ A hanseníase tem tratamento? } & Não sabe & 41 & 20,60 \\
\hline & Sabe & 158 & 79,40 \\
\hline \multirow[t]{2}{*}{ A hanseníase tem cura? } & Não sabe & 77 & 38,69 \\
\hline & Sabe & 122 & 61,31 \\
\hline \multirow{2}{*}{ Você foi vacinado? } & Não sabe & 177 & 88,94 \\
\hline & Sabe & 22 & 11,06 \\
\hline \multirow{2}{*}{ Existe vacina para a hanseníase? } & Não sabe & 153 & 76,88 \\
\hline & Sabe & 46 & 23,12 \\
\hline \multirow{2}{*}{$\begin{array}{l}\text { Você já viu alguma campanha/palestra } \\
\text { sobre a hanseníase? }\end{array}$} & Não sabe & 67 & 33,67 \\
\hline & Sabe & 132 & 66,33 \\
\hline \multicolumn{4}{|l|}{$\begin{array}{l}\text { Nível de conhecimento sobre a } \\
\text { hanseníase }\end{array}$} \\
\hline Conhece & & 3 & 1,51 \\
\hline Conhece pouco & & 79 & 39,70 \\
\hline Não conhece & & 117 & 58,79 \\
\hline
\end{tabular}

Fonte: Dados da pesquisa

Tabela 2 - Classificação do nível de conhecimento sobre a hanseníase dos doadores de sangue $(n=199)$ em relação ao gênero, faixa etária, nível de escolaridade, nível socioeconômico, estado civil e religião em frequência percentual (\%).

\begin{tabular}{|c|c|c|c|c|}
\hline & \multicolumn{3}{|c|}{ Nível de conhecimento } & \multirow{2}{*}{$\begin{array}{l}\text { Valor } \\
\text { de } p\end{array}$} \\
\hline & Conhece & $\begin{array}{l}\text { Conhece } \\
\text { pouco }\end{array}$ & $\begin{array}{c}\text { Não } \\
\text { conhece }\end{array}$ & \\
\hline \multicolumn{5}{|l|}{ Gênero } \\
\hline Feminino $(n=66)$ & 3,03 & 33,33 & 63,64 & \multirow{2}{*}{0,2431} \\
\hline Masculino $(n=133)$ & 0,75 & 42,86 & 56,39 & \\
\hline \multicolumn{5}{|l|}{ Faixa etária (anos) } \\
\hline $\begin{array}{l}\text { Jovem }(18-24) \\
(n=52)\end{array}$ & 7,69 & 36,54 & 55,77 & \multirow{3}{*}{0,2318} \\
\hline $\begin{array}{l}\text { Adulto Jovem } \\
(25-34)(n=65)\end{array}$ & 24,62 & 58,46 & 16,92 & \\
\hline $\begin{array}{l}\text { Adulto (35-49) } \\
(\mathrm{n}=64)\end{array}$ & 23,44 & 64,06 & 12,5 & \\
\hline
\end{tabular}




\begin{tabular}{|c|c|c|c|c|}
\hline & \multicolumn{3}{|c|}{ Nível de conhecimento } & \multirow{2}{*}{$\begin{array}{l}\text { Valor } \\
\text { de } p\end{array}$} \\
\hline & Conhece & $\begin{array}{l}\text { Conhece } \\
\text { pouco }\end{array}$ & $\begin{array}{c}\text { Não } \\
\text { conhece }\end{array}$ & \\
\hline $\begin{array}{l}\text { Adulto Maduro } \\
(50-64)(n=18)\end{array}$ & 38,89 & 55,56 & 5,55 & \\
\hline \multicolumn{5}{|l|}{$\begin{array}{l}\text { Nível de } \\
\text { escolaridade }\end{array}$} \\
\hline Fundamental (n=29) & 0,00 & 27,59 & 72,41 & \multirow{3}{*}{0,0273} \\
\hline Médio $(n=85)$ & 3,53 & 34,12 & 62,35 & \\
\hline Superior $(n=85)$ & 0,00 & 49,41 & 50,59 & \\
\hline \multicolumn{5}{|l|}{$\begin{array}{l}\text { Nível } \\
\text { socioeconômico }\end{array}$} \\
\hline$A(n=14)$ & 0,00 & 64,29 & 35,71 & \multirow{4}{*}{0,0894} \\
\hline$B(n=114)$ & 1,75 & 44,74 & 53,51 & \\
\hline$C(n=70)$ & 1,43 & 27,14 & 71,43 & \\
\hline$D(n=1)$ & 0,00 & 0,00 & 100,00 & \\
\hline \multicolumn{5}{|l|}{ Estado civil } \\
\hline Casado (n=109) & 0,92 & 44,95 & 54,13 & \multirow{3}{*}{0,4665} \\
\hline Divorciado ( $n=9$ ) & 0,00 & 33,33 & 66,67 & \\
\hline Solteiro $(n=81)$ & 2,47 & 33.33 & 64,20 & \\
\hline \multicolumn{5}{|l|}{ Religião } \\
\hline Católica (n=141) & 2,13 & 39,01 & 58,87 & \multirow{4}{*}{0,2328} \\
\hline Evangélica ( $n=45$ ) & 0,00 & 40,00 & 60,00 & \\
\hline Outras $(n=8)$ & 0,00 & 50,00 & 50,00 & \\
\hline Nenhuma $(n=2)$ & 0,00 & 50,00 & 50,00 & \\
\hline
\end{tabular}

Fonte: Dados da pesquisa

Dentre os participantes entrevistados, houve predomínio do sexo masculino, de adultos jovens e adultos, do ensino médio e superior, da classe $B$ e de religião católica. A distribuição de casados e solteiros demonstrou-se equivalente (Tabela 2). Após a avalição do questionário sobre o conhecimento da hanseníase, verificou-se que apenas o nível de escolaridade apresentou-se significativo em relação ao conhecimento da hanseníase $(p=0,0273)$ (Tabela 2).

\section{DISCUSSÃO}

A sua maioria dos participantes foi do sexo masculino, podendo estar associado ao fato de mulheres ficarem impossibilitadas de doar sangue, devido à critérios de exclusão como ciclo menstrual ou o período de amamentação. ${ }^{(13)}$ Contudo as mulheres apresentaram um melhor entendimento da hanseníase em relação aos homens, provavelmente por serem mais vigilantes quando se refere à saúde pessoal. ${ }^{(14)}$

Os doadores considerados jovens demonstraram pouco conhecimento em relação aos indivíduos com mais idade. Existem evidências que nesta fase os jovens, embora saudáveis, disponham menos entusiasmo pela escola, pois eles estão descobrindo ocupações mais atraentes do que estudar. ${ }^{(15)}$

Verificamos que houve uma distribuição relativamente próxima, entre o número de indivíduos solteiros e casados. Outros estudos com doadores de sangue apresentaram resultados com o predomínio de solteiros ou com predomínio de indivíduos que possuíam algum tipo de união estável. ${ }^{(16)}$

Verificamos que os doadores de sangue demonstraram regular conhecimento, independente da classe social. A condição socioeconômica influencia a ampliação da hanseníase e exibe uma associação direta com as condições precárias de moradia, baixa escolaridade, e ainda, com processos migratórios, os quais favorecem a propagação da doença e a sua distribuição espacial em locais de miséria. ${ }^{(6)}$

A maioria dos participantes não sabia o que é a hanseníase e alguns correlacionaram com a "doença do rato". Quanto à transmissão, os participantes relataram que a forma mais provável de era através do contato, entretanto, também obtivemos respostas errôneas, como, transmissão pela saliva, ar, sol, mosquito palha, por vírus, fezes, urina do rato, entre outras. As respostas erradas em relação ao mecanismo de transmissão verificadas no presente estudo, também, foram relatadas por Cavalieri e Grynszpan(17). Entretanto, entre as respostas encontradas por esses autores, estavam as transmissões da doença pelo ar, vias sexuais, hereditariedade, transfusão de sangue, uso de drogas injetáveis e falta de higiene. ${ }^{(17)}$

Os participantes do estudo, em sua maioria, se referiam de forma correta aos sintomas da doença, como manchas na pele e perda de sensibilidade, discordando de outro estudo, onde seus participantes frequentemente se referiam de forma isolada aos sinais e sintomas ou associavam a doença a fatores etiológicos não correspondentes. ${ }^{(18)}$

Em sua maioria, os participantes responderam que a hanseníase tinha cura, entretanto, alguns apresentaram dúvida, talvez pelo fato da doença deixar muitas sequelas, além da falta de adesão ao tratamento. Segundo dados do Ministério da Saúde, em 2011, a avaliação do grau de incapacidade na cura foi de $72,9 \%$, o que é considerado precário, refletindo o diagnóstico tardio da doença. ${ }^{(19)}$

Apesar das políticas de controle no Brasil, o coeficiente de detecção de casos novos de hanseníase não diminuiu. Essa situação pode ser decorrente do baixo nível de conhecimento sobre a doença, ponto de vulnerabilidade detectado tanto neste trabalho quanto em outros estudos. ${ }^{(19,20)}$ Estudo que avaliou o conhecimento da hanseníase antes e após uma ação educativa em unidade básica de saúde revelou um aumento significativo no número de acertos após a ação, confirmando a importância dos profissionais da saúde em realizar ações preventivas, promocionais e medidas educativas com o intuito de ampliar o conhecimento da população em geral. ${ }^{(9)}$

Percebe-se, então, que muitos já ouviram falar sobre a doença, mas não sabem dizer como se adquire, sugerindo que um dos maiores obstáculos para erradicação é o desconhecimento. Segundo as Diretrizes do Ministério da Saúde(21), a educação em saúde é uma das ações para a redução da carga da hanseníase no Brasil, e, a divulgação de atualizações sobre a doença deve ser uma 
ação colaborativa das três esferas governamentais com instituições e entidades da sociedade civil. (21)

Estratégias preventivas com o propósito de estender o conhecimento são fatores indispensáveis e o grande desafio é tornar a informação acessível, com linguagem adequada a qualquer cidadão.

\section{CONCLUSÃO}

A partir do presente estudo constatou-se um baixo nível de conhecimento da maioria dos doadores de sangue entrevistados sobre a forma de transmissão, tratamento e fontes de informação sobre a hanseníase. Diante dos resultados obtidos é evidente a necessidade da disseminação de informações referentes à doença, tendo em vista que esta se insere entre um dos principais problemas de saúde pública do país. Sugerimos um aprimoramento da divulgação das informações quanto à doença, através de campanhas com mídias que possam atingir diferentes grupos demográficos, a fim de promover melhorias nos serviços de saúde, acompanhamento dos doentes e prevenção da população saudável.

\section{REFERÊNCIAS}

1. BRASIL. Ministério da Saúde. Secretaria de Políticas de Saúde. Departamento de Atenção Básica. Guia para o Controle da hanseníase. Brasília: Ministério da Saúde, 2002. Disponível em: http://bvsms.saude. gov.br/bvs/publicacoes/guia_de_hanseniase.pdf. Acesso em: 01 ago. 2019.

2. BRAKEL, W. H. Van. et al. Disability in people affected by leprosy: the role of impairment, activity, social participation, stigma and discrimination. Glob Health Action, [s.I], v. 5, p. 18394. 2012.

3. WORLD HEALTH ORGANIZATION. Department of Control of Neglected Tropical Diseases. Global leprosy update, 2014: need for early case detection. Wkly Epidemiol., Rec., Geneve, v. 36. p. 90, 461476, 2015. Disponível em: http://www.who.int/wer/2015/wer9036. pdf?ua=1. Acesso em: 01 aug. 2019.

4. BRASIL. Governo do Brasil. Organização mundial elogia Brasil no combate de doenças. 2017. Disponível em: http://www.brasil.gov. br/editoria/saude/2014/04/organizacao-mundial-elogia-brasil-nocombate-de-doencas. Acesso em: 01 ago. 2019.

5. BRASIL. Ministério da Saúde. Sistema de Informações Hospitalares do SUS (SIH/SUS). 2016. Disponível em: http://tabnet.datasus.gov.br/ cgi/tabcgi.exe?sih/cnv/niuf.def. Acesso em: 01 ago. 2019.

6. QUEIROZ, M. S.; PUNTEL, M. A. A situação epidemiológica da hanseníase no Brasil e em Campinas. Rio de Janeiro: Editora FIOCRUZ, 1997.

7. BRASIL. Secretaria da Saúde. Hanseníase. Disponível em: http://www. saude.pr.gov.br/modules/conteudo/conteudo.php?conteudo=2799. Acesso em: 01 ago. 2019.
8. MARTINS-MELO F. R. et al. Mortality from neglected tropical diseases in Brazil. Bull World Health Organ, Geneve, v. 94, p. 103-110, Feb. 2016.

9. MOREIRA, A. J. et al. de. Ação educativa sobre hanseníase na população usuária das unidades básicas de saúde de Uberaba-MG. Saúde Debate, Rio de Janeiro, v. 38, n. 101, p. 234-243, jun. 2014.

10. ASSOCIAÇÃO BRASILEIRA DE EMPRESAS DE PESQUISA. Critério de Classificação do Brasil. Ibope [Internet]. 2014. Disponível em: http:// www.abep.org/. Acesso em: 10 fev. 2015.

11. CAMARGO, B. V. et al. Representações sociais do envelhecimento entre diferentes gerações no Brasil e na Itália. Psicol. pesq., Juiz de Fora, v. 8, n. 2, p. 179-188, dez. 2014.

12. FURTADO, M. L. C. Aplicativo computacional para a análise de experimentos envolvendo variáveis respostas categorizadas. 2003. 51 f. Dissertação (Mestrado em Agronomia) - Faculdade de Ciências Agronômicas da UNESP - Campus de Botucatu, 2003.

13. BRASIL. Ministerio da Saúde. Segurança transfuncional: um olhar sobre os serviços de hematoterapia das regiões Norte e Centro Oeste do Brasil. Brasilia, 2012.

14. SILVA, P. L. N. Perfil de conhecimentos sobre hanseníase entre moradores de uma Estratégia Saúde da Família. Hansen Int., Bauru, v. 37, n. 2, p. 31-39, 2012

15. ZAGURY, T. Encurtando a adolescência: orientações para pais e educadores. Rio de Janeiro: Record, 1999.

16. BRENER, S.; CAIAFFA, W. T.; PROIETTI, F. A. Fatores associados à aptidão clínica para a doação de sangue - determinantes demográficos e socioeconômicos. Rev. Bras. Hematol. Hemoter., São José do Rio Preto, v. 30 , n. 2, p. 108-113, abr. 2008.

17. CAVALIERE, I. A. L.; GRYNSZPAN, D. Fábrica de imaginário, usina de estigmas: conhecimento e crenças de uma comunidade escolar sobre hanseníase. Cad. Saúde Colet., Rio de Janeiro, v. 16, n. 2, p. 345-362. 2008.

18. GOMES, F. C. et al. Conhecimento usuário da atenção primária à saúde acerca da Hanseníase. Rev. Enferm., Recife, v. 8, n. 2, p. 36693676, out. 2014.

19. RODRIGUES, C. C. et al. Análise dos conhecimentos a respeito da hanseníase em academicos de medicina. Braz. J. Surg. Clin. Res., v. 4, n. 1, p. 23-27, set-nov. 2013.

20. DIAS, A.; CYRINO, E. G.; LASTÓRIA, J. C. Conhecimentos e necessidades de aprendizagem de estudantes de fisioterapia sobre a hanseníase. Hansen Int., Bauru, v. 32, n. 1, p. 9-18. 2007.

21. BRASIL. Ministério da Saúde. Diretrizes para vigilância, atenção e eliminação da Hanseníase como problema de saúde pública: manual técnico-operacional. Brasília, 2016.

Submetido em: $15 / 10 / 2019$

Aceito em: 07/02/2020 